\title{
Journal of Bacteriology and

\section{Proteomic Patterns in Serum and Urine of Patients Infected with Schistosoma mansoni: Basis for New Biomarker}

\author{
Yasser Elsherif ${ }^{\prime 2 *}$, El-Sayed Tharwa ${ }^{2}$, Gamal Badra ${ }^{2}$, Soraya Sharaf ${ }^{2}$, Mohsen Salama ${ }^{2}$, Imam Waked ${ }^{2}$ and Mark Thursz \\ 'Imperial College London, UK \\ ${ }^{2}$ National Liver Institute, Menoufia University, Egypt \\ *Corresponding author: Yasser Elsherif, Imperial College London, UK, Tel: +44(0)7883410748; E-mail: yassherif@hotmail.com \\ Received date: Sept 23, 2016; Accepted date: Oct 17, 2016; Published date: Oct 21, 2016
}

Copyright: @ 2016 Elsherif $\mathrm{Y}$, et al. This is an open-access article distributed under the terms of the Creative Commons Attribution License, which permits unrestricted use, distribution, and reproduction in any medium, provided the original author and source are credited.

\begin{abstract}
Diagnosis of $S$. mansoni infection is carried out by the detection of eggs in faeces. This method has low sensitivity, especially with patients in the acute phase of the illness or with low-intensity infection. Serological tests cannot differentiate between active and past infection. Non-invasive diagnostic tests for active disease and monitoring response to treatment are required. This study was performed to identify serum and urine proteomic based biomarkers in patients with $S$. mansoni infection before and after therapeutic intervention using Surface Enhanced Laser Desorption/lonization Time of Fight-Mass Spectrometry (SELDI TOF-MS). Serum samples were collected from 30 patients and urine samples from another15 patients both prior to and four to six weeks after treatment with praziquantel. All patients had a confirmed diagnosis of active infection on rectal biopsy. Serum and urine proteomic profiles were obtained by SELDI TOF-MS using cation capture (CM10) and immobilized metal affinity (IMAC30) ProteinChip ${ }^{\mathrm{TM}}$ arrays. In urine samples, nine protein peaks demonstrated significant differences between pre and post treatment samples: 46 kDa, 44 kDa, 34 KDa, 13.3 KDa, 10.8 KDa, 19.7 kDa, 15.9 kDa, 18.1 $\mathrm{kDa}, 4.7 \mathrm{KDa}$ (All had $\mathrm{p}<0.05$ ). On ROC curve analysis, only the protein at $4.7 \mathrm{kDa}$ showed a significant diagnostic signal $(A U R O C=0.77$ ) indicating that it has potential as biomarker for active infection. In serum samples, only four peaks were found to be significantly different between pre and post treatment groups ( $p$ value $<0.01$ ). However no serum peak demonstrated significance on ROC curve analysis. These results suggest that urinary proteomic testing may provide a non-invasive diagnostic test for S. mansoni.
\end{abstract}

Keywords: Surface-enhanced laser desorption/ionization time-offlight mass spectrometry (SELDI TOF-MS) technology; Schistosoma mansoni; Rectal biopsy

\section{Introduction}

Schistosomiasis is a parasitic disease caused by infection with the helminth Schistosoma species. Two hundred million people are infected worldwide, primarily in the equatorial areas Schistosoma mansoni ( $S$. mansoni) has been one of the major causes of liver diseases and portal hypertension related fatalities in many countries [1].

Diagnosis is pivotal to all aspects of schistosomiasis for assessment of morbidity, evaluation of chemotherapy and control measures. In spite of the generation of new knowledge in the area of $S$. mansoni, there remains the unsolved controversy associated with the basic diagnosis of this serious parasitic disease. This relates to the direct microscopic examination of faeces used for eggs detection. Stool examination has been and remains the main diagnostic method in use. Although it is a simple and straight forward technique, this method of diagnosis has variable sensitivity, especially in the presence of low intensity infection and in patients during the acute phase of the infection [2,3]. Furthermore, the low number of eggs in some cases and their fluctuating excretion make the diagnosis of many cases difficult. In a preliminary survey in our institute, 453 patients were screened for $S$. mansoni. Out of all patients, only 23/453 (5.1\%) were positive by stool examination while $169 / 453(37.3 \%)$ were positive by rectal biopsy. Furthermore, the low number of eggs in some cases and their fluctuating excretion make the diagnosis of many cases difficult.

Serological tests are not efficient to follow response to treatment. Schistosomal antibody detection assays, though sensitive, they do not however differentiate between active and past infection or even intensity of the infection [4]. It was anticipated that the difficulties associated with parasitological diagnosis might be overcome by antigen detection. However, this has been found unlikely. Because of the shared carbohydrate epitopes, schistosome- derived circulating antigens are not species specific [5]. Therefore this methodology can cross react with other infections species. In non-endemic areas, the diagnostic specificity is high because little false-positivity is found [6].

This study was designed to initiate a basis for easier, safer and a less invasive biomarker for detection of $S$. mansoni infection and treatment follow up. This was performed through protein profiling technology using the surface- enhanced laser desorption/ionization time-of-flight mass spectrometry (SELDI TOF-MS) technology.

\section{Patients and Methods}

Blood samples from 30 patients and mid-stream urine samples from another 15 patients were collected both prior to and four to six weeks after treatment with praziquantel $40 \mathrm{mg} / \mathrm{kg}$. Age ranged from 19 to 56 years (Mean=39.9), 76.7\% male. All patients had rectal biopsies to confirm active infection and infection clearance. All samples were collected in the endoscopy unit of the National Liver Institute within two hours after positive rectal snip. All patients came from rural areas in Delta region of Lower Egypt. Patients with any history liver or 
Page 2 of 4

kidney diseases, any known chronic illness other than Schistosomiasis were excluded. Blood samples were taken using similar $10 \mathrm{~cm}$ plastic syringes with the patient is sitting in upright position using a tourniquet. Samples were left at room temperature for one to two hours before spinning to extract serum by centrifuging the whole blood sample at $1000-1500 \mathrm{~g}$ for $10 \mathrm{~min}$ at $20-25^{\circ} \mathrm{C}$. The resulting supernatant was then aliquoted into Eppendorf tubes and immediately frozen at $-70^{\circ} \mathrm{C}$ until analysis. Urine samples were collected in sterile plastic tubes and kept in fridge $4^{\circ} \mathrm{C}$ for two to three hours before being transferred directly to $-70^{\circ} \mathrm{C}$ freezers in National Liver Institute of Egypt. Praziquental $40 \mathrm{mg} / \mathrm{kg}$ was given to every patient the same day and serum/urine samples were re-collected and frozen after four weeks with similar technique.

After collection of all samples, they were transferred for proteomic analysis. Samples have been taken from $-70^{\circ} \mathrm{C}$ freezers and put immediately in dry ice box then transferred directly from National Liver Institute (NLI) of Egypt to Imperial College London (ICL), UK where sample directly re-transferred to $-70^{\circ} \mathrm{C}$ freezers without thawing at any point. The overall journey from NLI freezer to ICL freezer took 10 hours. Samples were kept in $-70^{\circ} \mathrm{C}$ freezers until SELDI TOF protein profiling.

The study was approved by the Ethical Committee of the National Liver Institute, Menoufiya University according to the Declaration of Helsinki. All patients signed informed consent form prior to starting the study.

\section{SELDI-TOF Mass Spectrometry}

Serum and urine proteomic profiles were obtained by SELDI TOFMS using cation exchange surface (CM10) and immobilized metal affinity (IMAC30) ProteinChip ${ }^{\text {tu }}$ arrays (BioRad) to increase the chance of capturing different protein subsets on the two different chip surfaces. All samples have been performed under both high and low molecular weight settings as recommended by manufacturer. Pre and post treatment proteomic profiles were compared for any peak difference.

\section{Sample preparation}

Serum: Pre and post serum samples from $30 \mathrm{~S}$. mansoni infected patients (total of 60 serum samples) were studied. Samples were thawed on ice and aliquoted in the safety cabinet in two 96 wells' plates $(5 \mu \mathrm{l}$ for IMAC 30 and $10 \mu \mathrm{l}$ for CM10). Four samples were added: 2 control serum samples, 1 high molecular weight (HMW) standard and 1 low molecular weight (LMW) standard. Samples have been managed according to protocol standards.

Urine: Pre and post serum samples from 15 S. mansoni infected patients (total of 30 serum samples). Samples have been analysed for two types on ProteinChips CM10 and IMAC 30. Samples were thawed on ice and centrifuged at $7000 \mathrm{rpm}, 4^{\circ} \mathrm{C}$ for 5 minutes to remove any debris. Protein concentrations were determined using Bradford technique to determine protein concentrations and correction of high protein level variation in urine sample. Samples were aliquoted at 5 $\mathrm{ug} / \mathrm{ml}$ protein content of urine for both CM10 and IMAC $(5 \mu \mathrm{g} / \mathrm{ml}$ chosen after 5 samples trial on $5 \& 10 \mu \mathrm{g} / \mathrm{ml}$ concentrations). Two samples for one patients, pre and post treatment, were excluded as they were very dilute and needed larger amount of urine to achieve $5 \mu \mathrm{g}$ protein content. Total number of samples remained for testing was 28 . Samples were aliquoted in the safety cabinet in two 96 wells' plates ( 5 $\mu \mathrm{l}$ for IMAC 30 and $10 \mu \mathrm{l}$ for CM10).

\section{Protein array preparation}

\section{SELDI TOF-MS settings}

Time-of-flight spectra were generated using a PCS-4000 mass spectrometer (BioRad). For serum, spectra for the CM10ProteinChip were obtained at laser energy of 1500 and $2500 \mathrm{~nJ}$ (two warming shot of 1650 and $2750 \mathrm{~nJ}$ ); a focus mass of 6000 and $30000 \mathrm{Da}$; and the matrix attenuated to 1000 and 10000, for LMW and HMW settings respectively. Spectra for IMAC 30 were obtained at laser energy of 1400 and $3000 \mathrm{~nJ}$ (two warming shots of 1540 and $2750 \mathrm{~nJ}$ ); a focus mass of 6000 and 30000; and the matrix attenuated to 1000 and 10000 Da, for LMW and HMW settings respectively. Ten shots were obtained per position.

For urine samples, CM10 ProteinChip spectra were obtained at a laser energy of (1750-2400) and (1800-4400) nJ (Shots 110 collected in manual mode); a focus mass of 6000 and $30000 \mathrm{Da}$; and the matrix attenuated to 1000 and $10000 \mathrm{Da}$, for LMW and HMW settings respectively. Spectra for IMAC 30 were obtained at laser energy of 1400 and $3000 \mathrm{~nJ}$ (two warming shots of 1540 and $2750 \mathrm{~nJ}$ ); a focus mass of 6000 and $30000 \mathrm{Da}$; and the matrix attenuated to 1000 and 10000, for LMW and HMW settings respectively with 150 shots averaged per spot. Mass accuracy was calibrated externally using Allin-One Peptide or Protein molecular mass standards (BioRad).

\section{Peak detection, data analysis}

The Protein arrays were placed in a ProteinChip Reader Model PBS II and were read using ProteinChip Software, version 3.0.2 (Ciphergen Biosystems). Baseline subtraction was performed, followed by normalization of traces for intensity, using the instrument-dedicated software. Peaks were then detected using the built-in peak detection algorithm, setting the signal to noise ratio to five. Peaks below $2000 \mathrm{Da}$ were excluded to avoid noise from the energy absorbing matrix. Spectra between 1300 and 150,000 m/z were selected for analysis using Decision Trees.

\section{Results}

\section{Serum samples}

Pre and post treatment serum samples for 30 patients with active Schistosomiasis were studied, total 60 samples. Serum was chosen instead of plasma to avoid possible interaction between clotting factors in plasma and the sample binding surface. The sample collection procedure was stringent to prevent any possible artefact. Experiments were then carried out on CM10 and IMAC30.

Serum results of CM10 analysis at low molecular weight at LMW settings showed 76 peak clusters. Two peaks were significantly different between pre and post treatment levels $(\mathrm{p}<0.01)$; however, they had poor receiver operating curve (ROC) curves of 0.32 . At HMW settings, 61 clusters were found. Only 1 peak showed significant $\mathrm{p}$ values $(\mathrm{p}<0.01)$, but poor ROC curves of 0.31 .

In IMAC 30 at LMW settings, 93 peaks clusters were found. Only one had significantly different magnitude between pre and post treatment levels $(\mathrm{p}<0.01)$, with ROC curves of 0.68 . Serum results of IMAC30 at HMW setting, 23 peaks clusters were found but none reached statistical significance $(\mathrm{p}<0.01)$. 
Citation: Elsherif Y, Tharwa ES, Badra G, Sharaf S, Salama M, et al. (2016) Proteomic Patterns in Serum and Urine of Patients Infected with Schistosoma mansoni: Basis for New Biomarker. J Bacteriol Parasitol 7: 291. doi:10.4172/2155-9597.1000291

Page 3 of 4

\section{Urine results}

Pre and post treatment urine samples for 14 patients were analysed; total 28 samples. Urine proteomic profiles were obtained by SELDI TOF-MS using CM10 and IMAC30 ProteinChip ${ }^{\mathrm{Tm}}$ arrays. Unlike serum, the protein concentrations in urine samples vary widely. To avoid variation artefacts, protein concentrations were determined using Bradford protein assay. After trial and errors we used a urinary protein concentration of $5 \mu \mathrm{g} / \mathrm{ml}$ for each sample for both IMAC 30 and CM10 Chips to provide optimal signal: noise ratio. This protein concentration showed better spectra in both ProteinChips and most of samples gave good peaks.

Urine results of CM10 analysis at LMW setting revealed that the protein at $4.7 \mathrm{kDa}$ gave a significant diagnostic signal difference between pre and post treatment samples $(p=0.02)$ with a significant ROC curve $=0.77$, indicating that it has potential as biomarker for active infection. In HMW settings, 3 protein peaks at $46 \mathrm{kDa}, 44 \mathrm{kDa}$ and $34 \mathrm{kDa}$ showed significant differences between pre and post treatment values $(\mathrm{P}<0.04)$; however, these peaks were expressed at very low intensities to allow using them as biomarkers (Figures 1 and 2).

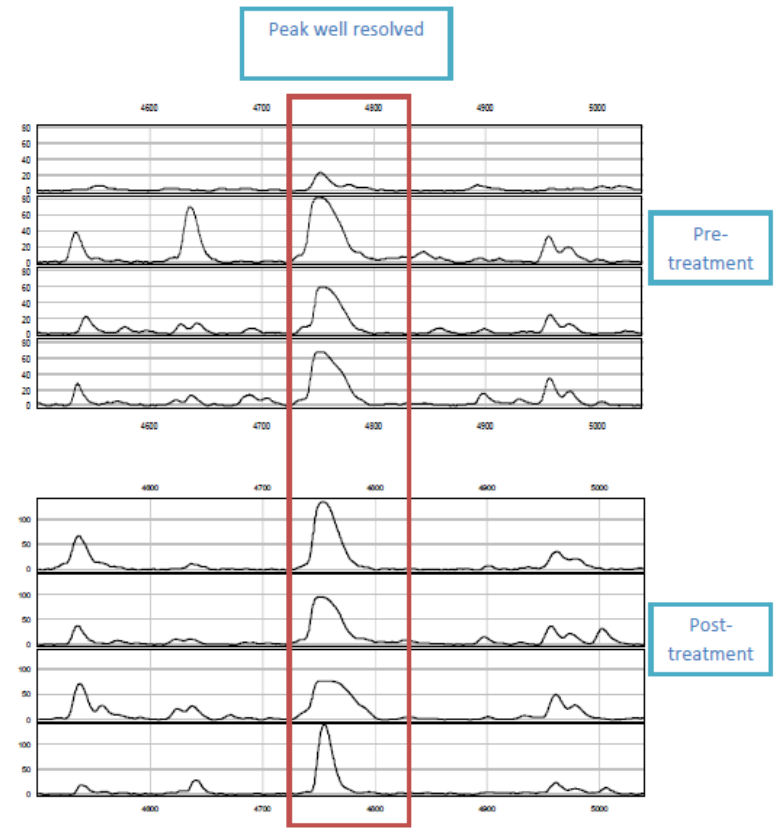

Figure 1: Urine results of CM10 PH4 at LMW setting: a protein of $4.7 \mathrm{kDa}$ has shown to give a significant diagnostic signal difference between pre and post treatment samples $(\mathrm{P}<0.05)$.

Analysis of urine samples by IMAC30 at LMW setting, many clusters were found, however only 2 peaks $(10.8 \mathrm{kDa} \& 18.3 \mathrm{kDa})$ were significant $(\mathrm{p}<0.05)$. These peaks had very low intensities with poor sensitivity or specificity. The second peak had a very low intensities so that the software had to estimate them indicating potentially poor performance as biomarkers (Figure 3).

Analysis of urine samples by IMAC 30 at HMW setting showed 3 significant peaks $(19.7 \mathrm{kDa}, \mathrm{p}=0.01 ; 15.9 \mathrm{kDa}, \mathrm{p}=0.01 ; 18.1 \mathrm{kDa}$, $\mathrm{p}=0.03$ ). Although significant differences were seen between pre and post treatment samples, they were expressed at very low levels making them unsuitable for biomarker purposes. For example, although the 19.7 kDa peak showed significant diagnostic difference with reasonable good ROC curve, the low levels of this protein would make identification technically challenging.
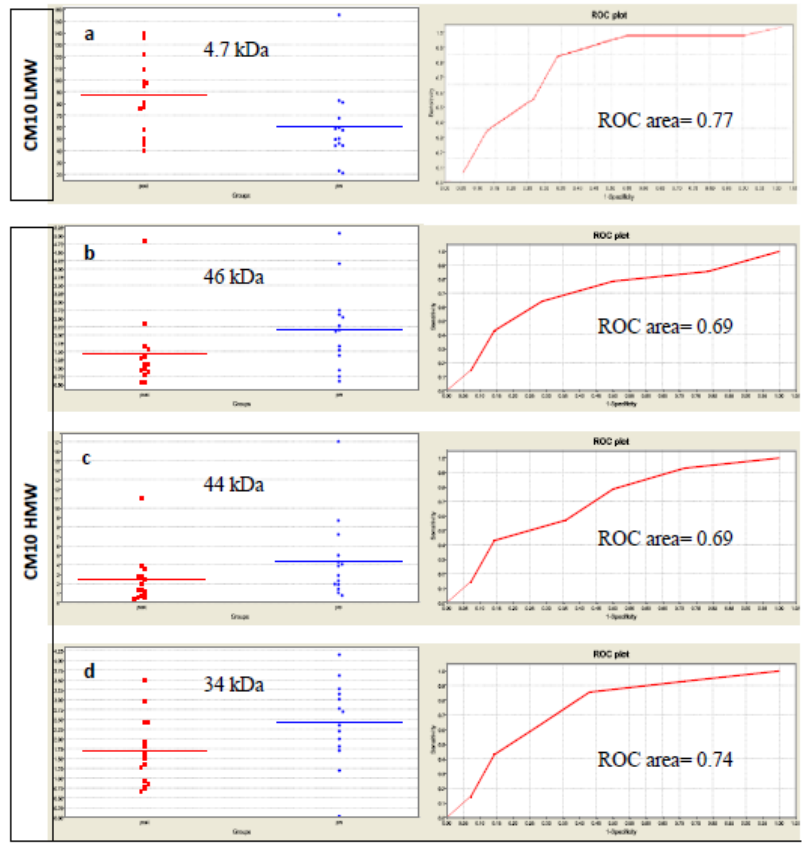

Figure 2: CM10 PH4 at LMW and HMW settings: CM10 PH4 at LMW setting revealed many peak clusters, however only 1 peak (a) $4.7 \mathrm{kDa}$ was significant at $\mathrm{P}=0.02$ with $\mathrm{ROC}$ curve of 0.77 . This protein is repressed during infection and showed a significant increase after drug treatment. Results of CM10 PH4 at HMW setting showed 3 peaks with $\mathrm{P}$ value $<0.04$ (b) $46 \mathrm{kDa}$, (c) $44 \mathrm{kDa}$ and (d) $34 \mathrm{kDa}$ peptide, however these peaks were expressed at very low intensities (see Y-axis).

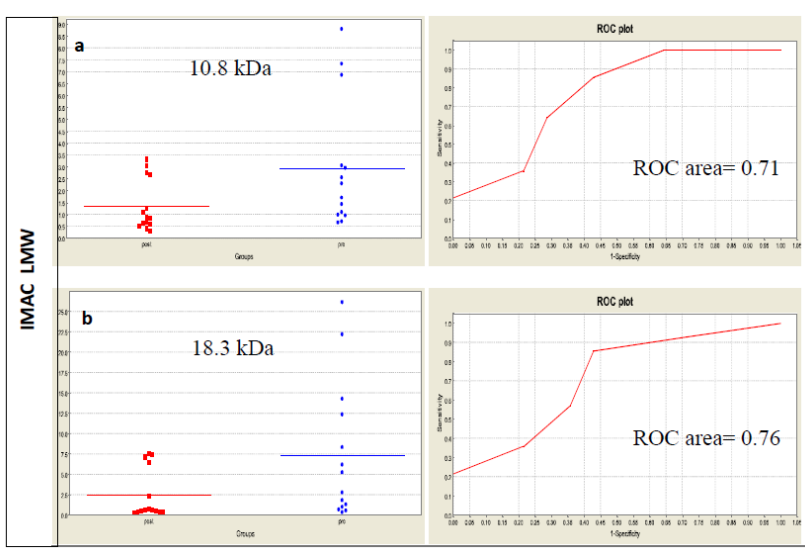

Figure 3: Results of IMAC 30 at LMW setting showed two significant peaks (a) $10.8 \mathrm{kDa}$, and (b) $18.3 \mathrm{kDa}(\mathrm{P}=0.03)$. These peaks had very low intensities to be considered as biomarkers. 


\section{Discussion}

This study holds promise for a novel diagnostic test with high sensitivity and specificity for improved disease surveillance and control. It was designed to screen proteins/peptides in serum and urine of $S$. mansoni infected patients before and after therapeutic intervention to identify a potential proteomic based biomarkers for diagnosis, monitoring of disease progression and the evaluation of chemotherapeutic interventions using Surface Enhanced Laser Desorption/Ionization Time of Fight-Mass Spectrometry technology.

All patients were recruited from similar background in Delta region of north Egypt to exclude environmental confounders. Patients were otherwise healthy with no evidence of viral hepatitis or other liver disease. All patients have been diagnosed by rectal biopsies and the standard treatment praziquantel was used. In serum samples, four protein peaks have been found to be significantly different between pre and post treatment groups $(\mathrm{p}<0.01)$, however these peaks showed poor ROC curves indicating poor sensitivity and specificity as biomarkers. In urine samples, Nine protein peaks demonstrated significant differences between pre and post treatment samples: $46 \mathrm{kDa}, 44 \mathrm{kDa}$, $34 \mathrm{KDa}, 13.3 \mathrm{KDa}, 10.8 \mathrm{KDa}, 19.7 \mathrm{kDa}, 15.9 \mathrm{kDa}, 18.1 \mathrm{kDa}, 4.7 \mathrm{KDa}$ (All $\mathrm{p}<0.05$ ). Out of them, the peak at $4.7 \mathrm{kDa}$ expressed the only protein that showed good ROC curve. This suggests that protein 4.7 $\mathrm{kDa}$ could be a novel biomarker. This protein was repressed during infection and revealed a significant increase after drug treatment (Figure 1)

Many proteomic based studies have been performed recently explored the urinary proteome for biomarkers of different diseases for screening, diagnosis and progression. Compared with other biological fluids, this approach has a number of practical advantages. For instance, it has been shown that proteins in serum and plasma may degrade rapidly when handled inappropriately; urinary proteins, on the contrary, remain stable to perform reliable proteome analysis [7].

On the other hand, unlike serum, urine contains very variable amounts of proteins. These changes can be present between patients and even for the same patient depending on time of sample collection (e.g. early morning, late afternoon or after physical exercise). This variation needs to be corrected before applying samples to the surface of ProteinChips to avoid result bias.

This study showed that many significant protein peaks between pre and post treatment in both serum and urine. The majority of them, however, showed poor ROC curves indicating poor biomarkers potentiality. The reason for these results could be the fact that those patients have light infection which may be reflected on their serum and urine biomarkers. Those patient were diagnosed with rectal biopsies, however all of them had negative microscopic stool analysis suggesting light infection. This may have caused the peaks to be significantly different between pre and post treatment groups, but not significant enough for optimal diagnostic performance on the ROC curve analysis. Other factors may influence the results. The follow up period of four weeks may not be enough to have a significant full picture of proteomic changes. We have chosen the 4 to 6 weeks follow up period based on previous study who suggested this period as the preferred timing after treatment [8]. In addition, longer follow up periods could carry higher risk of acquiring a new infection or losing follow up.

It was also noted that urine samples gave better potential as biomarkers than serum samples. Although this result may be a little confusing in regard of intestinal schistosmiasis, urine revealed better outcome as a source of biomarkers in $S$. mansoni in earlier studies. Antigen detection in serum was not sensitive in light infections and therefore less useful for clinical applications [9]. That is why it has been used mainly for research and for epidemiological and therapeutic studies. Urine based antigen detection assays had better potential than serum for the development reagent strips based biomarker development [10]. For this reason urine has been widely used for research in epidemiological and therapeutic studies.

In conclusion, these results suggest that urinary proteomic testing may provide a non-invasive diagnostic test for $S$. mansoni. Development of an ELISA based test will be needed to convert this into a practical diagnostic assay.

\section{References}

1. Gryseels B, Polman K, Clerinx J, Kestens L (2006) Human schistosomiasis. Lancet 368: 1106-1118.

2. Gryseels B, Nkulikyinka L (1988) The distribution of Schistosoma mansoni in the Rusizi plain (Burundi). Ann Trop Med Parasitol 82: 581-590.

3. Brindley PJ (2005) The molecular biology of schistosomes. Trends Parasitology 21: 533-536.

4. Mott PK, Dixon H (1982) Collaborative study on antigen for immunodiagnosis of schistosomiasis mansoni. Bull World Health Organ 63: 729-753.

5. Agnew A, Fulford AJ, De Jonge N, Krijger FW, Rodriguez-Chacon M, et al. (1995) The relationship between worm burden and levels of a circulating antigen (CAA) of 5 species of Schistosoma in mice. Parasitology 111: 67-76.

6. Van Lieshout L, Panday UG, De Jonge N, Krijger FW, Oostburg BF, et al. (1995) Immunodiagnosis of schistosomiasis mansoni in a low endemic area of Surinam by determination of the circulating antigens CAA and CCA. Acta Trop 59: 19-29.

7. Theodorescu D, Wittke S, Ross MM, Walden M, Conaway M, et al. (2006) Discovery and validation of new protein biomarkers for urothelial cancer: a prospective analysis. Lancet Oncol 7: 230-240.

8. Renganathan E, Cioli D (1998) An international initiative on praziquantel use. Trends in Parasitology. 14: 390-391.

9. van Lieshout L, Polderman AM, Visser LG, Verwey JJ, Deelder AM (1997) Detection of the circulating antigens CAA and CCA in a group of Dutch travellers with acute schistosomiasis. Trop Med Int Health 2: 551-557.

10. van Dam GJ, Wichers JH, Ferreira TM, Ghati D, van Amerongen A, et al. (2004) Diagnosis of schistosomiasis by reagent strip test for detection of circulating cathodic antigen. J Clin Microbiol 42: 5458-5461. 